\title{
In situ solid-state NMR and XRD studies of the ADOR process and the unusual structure of zeolite IPC- 6
}

Samuel A. Morris, ${ }^{1}$ Giulia P.M. Bignami, ${ }^{1}$ Yuyang Tian, ${ }^{1}$ Marta Navarro, ${ }^{1}$ Daniel S. Firth, ${ }^{1}$ Jiri Cejka, ${ }^{2,3}$ Paul S. Wheatley, ${ }^{1}$ Daniel M. Dawson, ${ }^{1}$ Wojciech A. Slawinski, ${ }^{4,5}$ David S. Wragg, ${ }^{4}$ Russell E. Morris, ${ }^{1,3}$ Sharon E. Ashbrook ${ }^{1} *$

${ }^{1}$ EaStCHEM School of Chemistry, University of St Andrews, St Andrews KY16 9ST, UK

${ }^{2}$ J. Heyrovský Institute of Physical Chemistry, Academy of Sciences of the Czech

Republic, v.v.i., Dolejškova 3, 18223 Prague 8, Czech Republic

${ }^{3}$ Department of Physical and Macromolecular Chemistry, Faculty of Sciences, Charles

University in Prague, Hlavova 8, 12843 Prague 2, Czech Republic

${ }^{4}$ Department of Chemistry, University of Oslo, Sem Sælandsvei 26, 0371 Oslo, Norway

${ }^{5}$ ISIS Facility, Rutherford Appleton Laboratory, Harwell Oxford, Didcot, Oxfordshire, OX11 0QX UK

The Assembly-Disassembly-Organisation-Reassembly (ADOR) mechanism is a recent method for preparing inorganic framework materials and, in particular, zeolites. This flexible approach has enabled the synthesis of isoreticular families of zeolites with unprecedented continuous control over porosity, and the design and preparation of materials that would have been difficult - or even impossible - to obtain using traditional hydrothermal techniques. Applying the ADOR process to a parent zeolite with the UTL framework topology, for example, has led to six previously unknown zeolites (named IPC- $n$ with $n=2,4,6,7,9$ and 10). To realize the full potential of the ADOR method, however, a further understanding of the complex mechanism at play is needed. Here, we probe the disassembly, organisation and reassembly steps of the ADOR process through a combination of in situ solid-state nuclear magnetic resonance (NMR) spectroscopy and powder Xray diffraction (PXRD) experiments. We further use the insight gained to explain the formation of the intriguing structure of zeolite IPC-6.

The recently-discovered ADOR process ${ }^{1-4}$ has proved to be effective for the preparation of new silicate and aluminosilicate zeolites, providing routes to 'unfeasible' synthesis targets with novel structural features ${ }^{3}$ and to families of isoreticular solids whose pore size can be precisely controlled over the whole range of zeolite porosity, from small pore all the way up to extra-large pore materials. ${ }^{1,4}$ The process comprises four distinct steps. The assembly (A) process involves the preparation of a parent zeolite with suitable chemical and topological properties for 
future manipulations. The key feature of the parent zeolites is the presence of a chemical 'weakness' in a specific structural site. ${ }^{5}$ Zeolites that have silica-rich layers linked by germanium-rich cubic units, termed double four rings $(d 4 r s)$, such as those with the UTL, ${ }^{6,7} \mathrm{IWW}^{8,9}$ and UOV ${ }^{10,11}$ topologies, are particularly suitable for the process. The $d 4 r$ units each comprise eight tetrahedra and contain approximately $50 \% \mathrm{Ge}$ and $50 \% \mathrm{Si}$.

Disassembly (D) of the parent zeolite through exposure to a suitable chemical stimulant, such as water or acid, can then lead to removal of the weaker units. In the case of UTL as a parent zeolite, exposure to aqueous acid solutions leads to removal of the $d 4 r$ units to leave a layered intermediate, called IPC-1P. ${ }^{12}$ The organisation (O) step then determines the final relative orientation of the intermediate layers and the subsequent reassembly (R) step traps this orientation into the final material. The fundamental difference between traditional hydrothermal synthesis and the ADOR process is that the final framework forming step in the former is a reversible crystallisation while that in the latter is an irreversible condensation. This opens up the possibility of making zeolites that would be avoided in hydrothermal synthesis, such as those with framework lattice energies that are higher than expected (i.e., the so called 'unfeasible' zeolites). ${ }^{3}$

One consequence of the organisation step is the possibility of using different structural units to link the layers to form the different zeolites, leading to isoreticular families. ${ }^{1,4}$ Controlling these linkages offers an important tool by which the final structures can be precisely tuned, and leads to, amongst other things, the possibility of continuously tuneable porosity. ${ }^{4}$ The isoreticular family of structures discussed in this manuscript are IPC-4, IPC-2 and IPC-6, which contain the same UTL layer types linked by oxygen atoms (which we call PCR linkages, as they are present in the PCR zeolite topology), single four ring units (called OKO linkages) and 50\%/50\% PCR/OKO linkages respectively. ${ }^{4}$ Here we report in situ magic angle spinning (MAS) solid-state NMR and XRD mechanistic studies on the evolution of the disassembly, organisation and reassembly steps in order to fully understand how these different interlayer linkages develop over the course of the reaction under the relevant conditions. We then use this information to explain how the process can form the surprising structure of IPC-6.

\section{Results and Discussion}

To complete time-resolved in situ solid-state MAS NMR experiments, it was necessary to isotopically enrich the parent UTL materials in ${ }^{29} \mathrm{Si}$ as described in the Materials and Methods section. Disassembly via hydrolysis of the parent ${ }^{29} \mathrm{Si}-$ enriched germanosilicate zeolite UTL was followed by in situ MAS NMR experiments, which show how the number of $\mathrm{Q}^{3}\left(\mathrm{O}_{3} \mathrm{Si}-\mathrm{OH}\right)$ silicon species grows relatively quickly, consistent with fast hydrolysis and removal of the $d 4 r$ units from between the layers to leave IPC-1P (Figure 1a). XRD also shows that this hydrolysis is complete relatively quickly (within 5 minutes) if a high volume $(40 \mathrm{~mL})$ of hydrolysis liquid is used, while it can be slowed down to several hours in low volumes $(<10 \mathrm{~mL}$ such as those used in the in situ NMR experiments). The outcome of the disassembly step is broadly the 
same no matter what the acidity of hydrolysis conditions, although it is more rapid in high acidity conditions.

While the disassembly step is common to all conditions, there are several different pathways that can be taken by the reaction once the organisation step begins, and it is this process that defines the overall outcome (Figure 2). In low acidic conditions (either neutral water or $0.1 \mathrm{M}$ aqueous $\mathrm{HCl}$ ) the IPC-1P layers are stable and do not evolve chemically even when heated up to $95^{\circ} \mathrm{C}$.

a

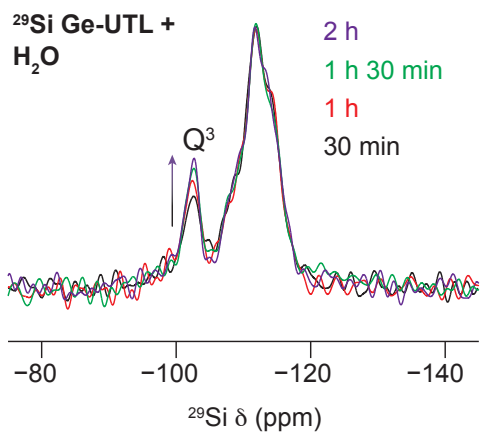

C

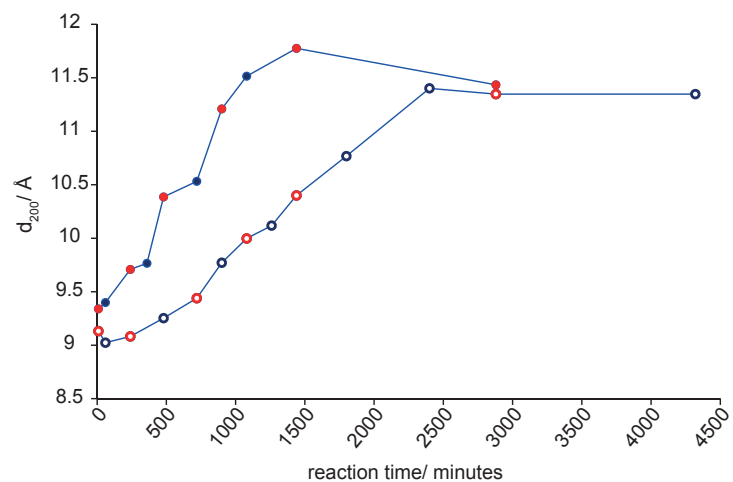

b
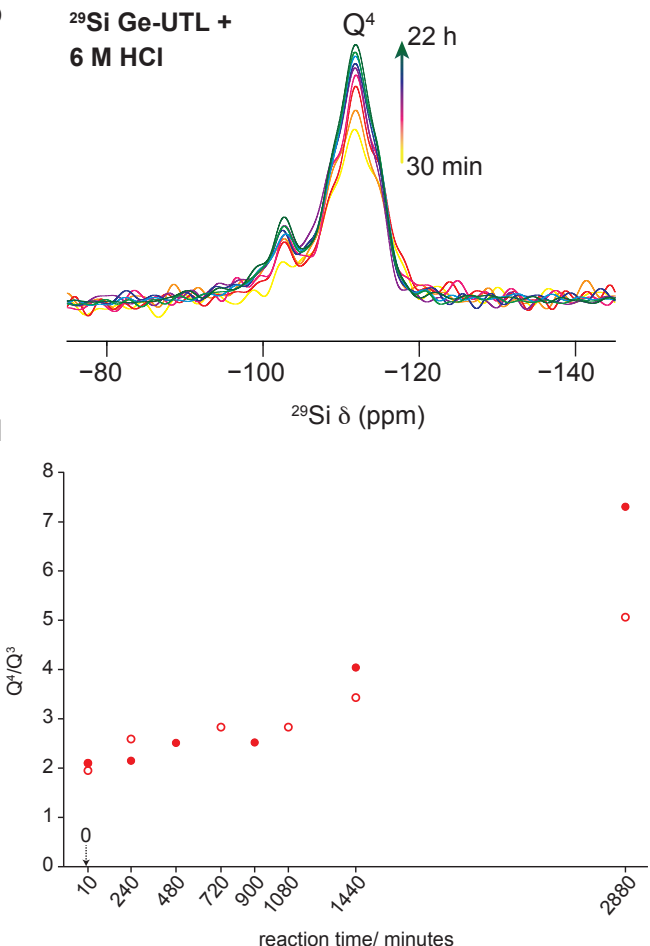

Figure 1. In situ NMR studies of the disassembly and organisation (rearrangement) steps in the ADOR process. (a) In situ ${ }^{29}$ Si MAS NMR spectra following the disassembly step of UTL in water, showing a marked increase in the amount of $\mathrm{Q}^{3}$ sites as the disassembly process is completed to form IPC-1P. (b) In situ ${ }^{29}$ Si MAS NMR of the organisation process taking place in 6 $\mathrm{M} \mathrm{HCl}$ showing how the amount of $\mathrm{Q}^{4}$ sites increases with time during the rearrangement, forming successively IPC-6P and then IPC-2P. Note that the extra silicon needed for this comes from reintercalation of Si originally removed from the $d 4 r$ units during the disassembly step (c) Plot of the interlayer peak d-spacing $\left(\mathrm{d}_{200}\right)$ from the ex situ PXRD patterns for the ADOR process applied to UTL under two different conditions (1.5 M, open circles, and $5 \mathrm{M} \mathrm{HCl}$, closed circles), showing a steady shift with time. The rate of shift is increased for the higher acidity (Note red data points indicate samples that, in their hydrolysed form, are also probed by the ex situ NMR experiments shown in panel (d)). (d) Plot of the $\mathrm{Q}^{4} / \mathrm{Q}^{3}$ ratio from the ex situ ${ }^{29} \mathrm{Si}$ MAS NMR spectra of the samples in panel (c), showing a faster increase with time for the samples treated with $5 \mathrm{M}$ acid (closed circles) compared with those treated with $1.5 \mathrm{M}$ (open circles).

In higher acidity conditions there is, however, a significant change in the chemistry of the system. In situ MAS NMR ${ }^{29}$ Si spectra show the same fast production of $Q^{3}$ sites on hydrolysis, followed by a significant relative increase in the intensity of the $\mathrm{Q}^{4}\left(\mathrm{Si}(\mathrm{OSi})_{4}\right)$ resonances over longer time periods (Figure $1 \mathrm{~b}$ ). This change in intensity is consistent with a rearrangement, such that the layers become reconnected by silica species. The $Q^{3}$ Si species formed by hydrolysis are converted to $Q^{4}$ species during this rearrangement by addition of extra Si between the layers. Note that the 
disassembly step has removed both germanium and silicon from the $d 4 r$ units between the layers to form IPC-1P, and so there is significant extra silicon in the system that is available for re-intercalation between the layers. Although the silicon in the solution is not directly observable in the in situ NMR experiment, conventional solution-state NMR analysis of the reaction solution recovered from ex situ hydrolysis of ${ }^{29} \mathrm{Si}$-enriched Ge-UTL shows the presence of silicon species in the solution.

Ex situ PXRD patterns of the calcined materials recovered after the higher acidity treatments (see Materials and Methods section) show a significant change in the position of the 200 reflection as a function of hydrolysis time (Figure 1c). The dspacing of the 200 reflection $\left(d_{200}\right)$ is, in essence, a measure of the average interlayer spacing in the material, and $2 \mathrm{~d}_{200}$ is a good measure of the repeat distance in the materials (Figures 2 and 3 ). As the silica rearrangement proceeds it will necessarily increase the average interlayer spacing (Figure 2). The $d$-spacing of this reflection increases steadily over time until a maximum is reached, before reducing slightly to give a final material we call IPC-2P that, when calcined, gives the zeolite IPC-2 (Figure 1c). Ex situ ${ }^{29} \mathrm{Si} N \mathrm{NMR}$ measurements of these samples prior to calcination show that the $Q^{4} / Q^{3}$ ratio changes with the amount of signal due to the $Q^{4}$ sites increasing over time relative to the $Q^{3}$ (Figure $1 d$ ), the same pattern of signal evolution as was seen in the in situ NMR described above. However, this signal is still evolving with time even when $d_{200}$ no longer changes. This indicates that changes to the local structure are ongoing, even when the final interlayer spacing has been reached. The reintercalation of silicon and rearrangement into the IPC-2P structure is clearly faster when higher acidity is used (Figures $1 \mathrm{c}$ and $1 \mathrm{~d}$ ). 


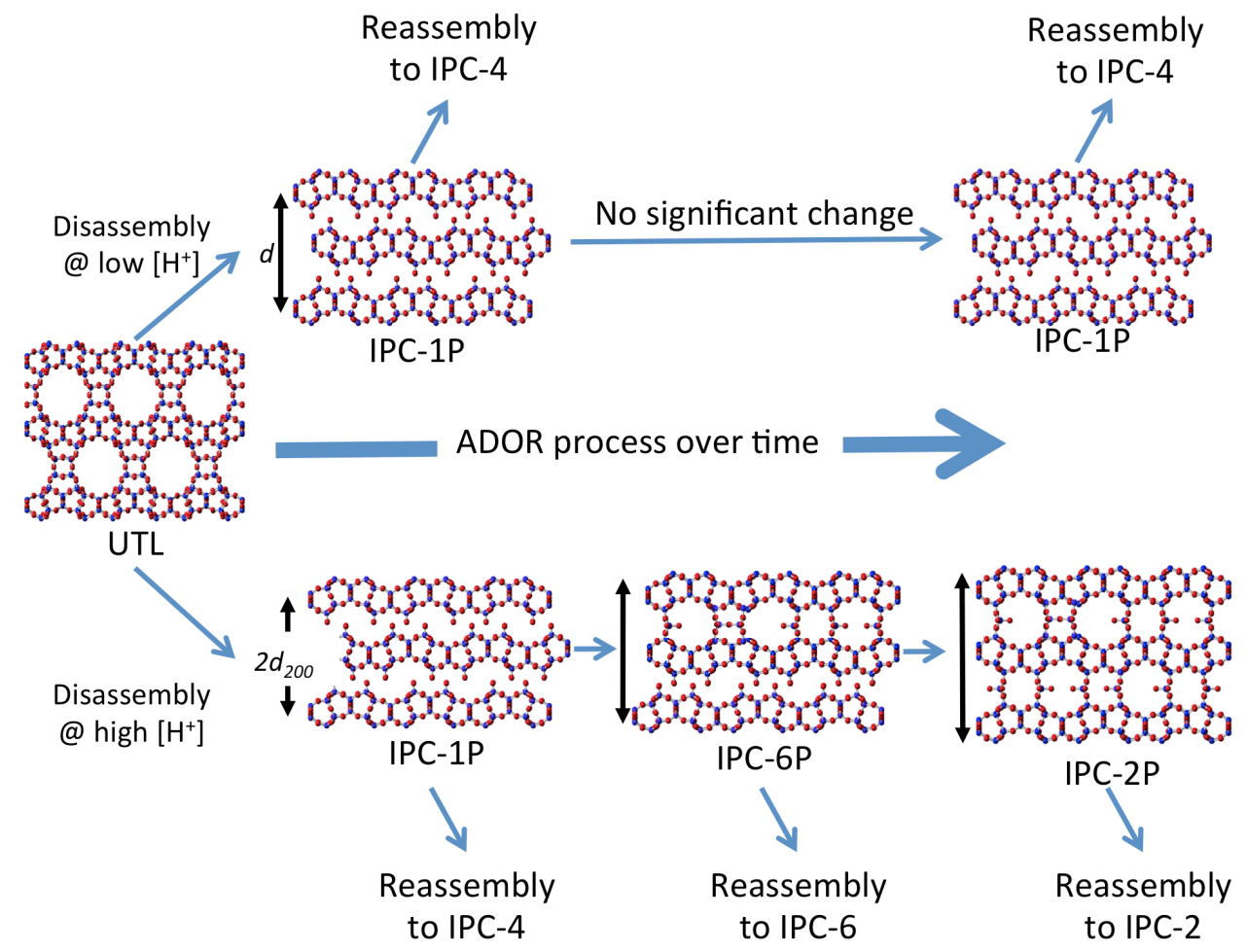

Figure 2. The mechanism of the ADOR process as revealed by the in situ studies. The mechanism of the ADOR process is shown under two limiting conditions. The top pathway shows the process under low acidity conditions, where the disassembly process removes the $d 4 r$ units from between the layers to leave IPC-1P. Low acidity conditions do not promote silica reintercalation/rearrangement and the only product on calcination is IPC-4, containing $100 \%$ PCR-type linkages. The bottom pathway shows how the disassembly process in high acidity conditions is essentially the same but that, with time, the rearrangement evolves to reintroduce silica into the interlayer space, connecting the layers. At intermediate times, only $50 \%$ of the layers are connected, leading to IPC-6 (50\% PCR, 50\% OKO connections) on calcination, while at longer times all the layers are connected leading to IPC-2 (with $100 \%$ OKO connections). In all figures, red spheres depict oxygen atoms and blue spheres represent silicon (or silicon or germanium in the case of UTL). 

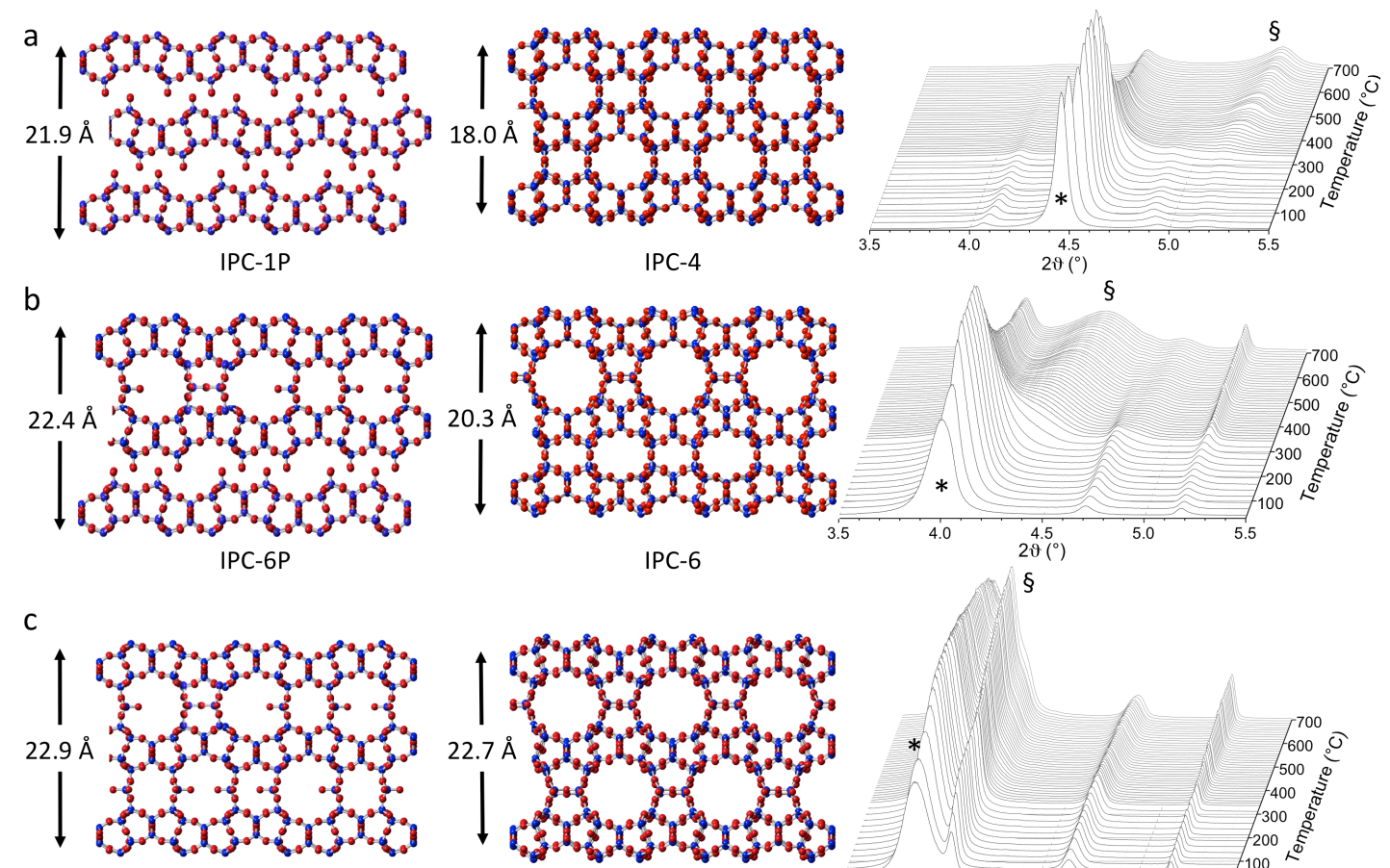

IPC-2P

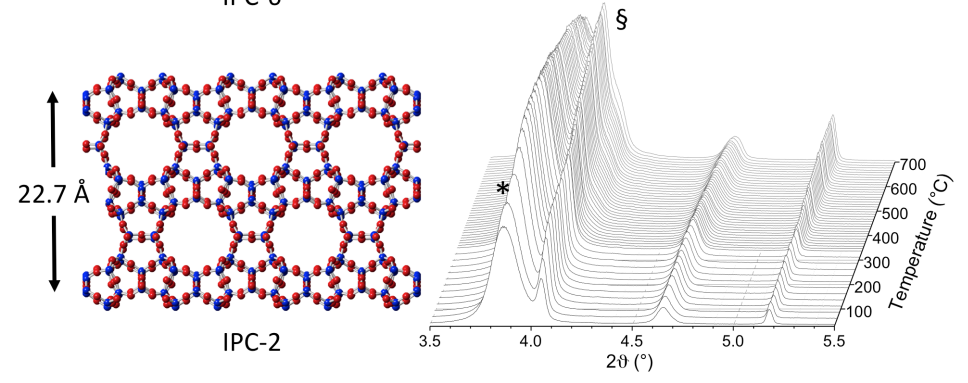

Figure 3. In situ XRD studies of the reassembly step of the ADOR process. (a) The reassembly of IPC-1P (left) to form IPC-4 (centre) and the corresponding in situ X-ray dffractogram showing how the materials change during this thermally-induced process. (b) The reassembly of IPC-6P to IPC- 6 and (c) the reassembly of IPC-2P to IPC-2. The value of $2 \mathrm{~d}_{200}$ is marked for each structure, with the position of the 200 reflection for IPC-1P, IPC-2P and IPC-6P marked by a * and the 200 reflection position for IPC-4, IPC-2 and IPC- 6 shown by a $\S$. Note that the difference in the 200 reflection position is large for IPC-1P and IPC-4, smaller for IPC-6P and IPC-6 and smaller still for IPC-2P and IPC-2.

All of the data presented above supports the presence of a silica rearrangement process that inserts silicon between the layers and increases the $Q^{4} / Q^{3}$ ratio. Figure 2 shows the proposed mechanism for the process as it evolves. There is strong evidence that as the rearrangement proceeds the layers become reconnected. IPC$1 \mathrm{P}$ can be easily swollen using, for example, CTAB-type surfactants. However, IPC-2P cannot be swollen at all. The mechanism can be further confirmed by using in situ synchrotron PXRD to probe the reassembly step (Figure 3). In these experiments, the precursor intermediates IPC-1P, IPC-6P and IPC-2P are heated from room temperature to $700{ }^{\circ} \mathrm{C}$, and the progress of the temperature-induced condensation to form the final materials is followed. Of particular importance are the positions of the 200 reflection which, as described above, give the average interlayer spacing in the material (Figure 3). On reassembly of IPC-1P (Figure 3a) the $d_{200}$-spacing reduces from $\sim 10.9 \AA$ to $\sim 9.0 \AA$ - the characteristic $\mathrm{d}_{200}$ for zeolite IPC-4. For IPC-2P, however, the $\mathrm{d}$-spacing is almost invariant on reassembly to IPC-2, changing only slightly from 11.5 to $11.4 \AA$. This adds further evidence that the layers are tied together by the silica species after the rearrangement process is complete, as shown in Figure 3c. The only change between IPC-2P and IPC- 2 is the completion of the condensation 
process, so that all (or at least the vast majority) of the silicon atoms end up as $Q^{4}$. In IPC-1P the layers are not tied by covalent linkages, which means that the reassembly condensation reaction decreases the interlayer spacing between each pair of layers (as IPC-4 has only PCR-type linkages). IPC-6 is intermediate between IPC-4 and IPC-2, having 50\% PCR linkages and 50\% OKO-type linkages. For IPC-6P the change in $\mathrm{d}_{200}$ spacing is from $\sim 11.2$ to $\sim 10.1 \AA$, consistent with the view that in IPC-6P $50 \%$ of the layers are tied together, but $50 \%$ are unconnected (Figure $3 \mathrm{~b}$ ). It is interesting to note that the rearrangement process described above leads to scrambling of the oxygen atoms, as can be shown using isotopically-enriched $\mathrm{H}_{2}{ }^{17} \mathrm{O}$ for the disassembly hydrolysis, as both $\mathrm{Si}-\mathrm{O}-\mathrm{Si}$ and $\mathrm{Si}-\mathrm{OH}$ oxygens are enriched as shown by ${ }^{17} \mathrm{O}$ MAS NMR spectra collected at 20.0 T (See supplementary information, Figure 1). This is an important result as it shows the process overall is highly dynamic, with the oxygen atoms from the hydrolysis water being incorporated throughout the material and not just in the interlayer regions as might be expected from a simplistic picture of the reaction.

The in situ studies very nicely describe how the interlayer spacings develop with time, but say nothing about how the connections between any two layers are related to those between other layers. IPC- 6 is a particularly intriguing structure as there are many different ways to arrange a 50:50 mixture of PCR- and OKO-type linkages. The interconnections between two layers are always all PCR or all OKO there is no evidence from any of the TEM studies for mixed PCR/OKO linkages between the same layers, and there is no evidence of the broadening in the PXRD patterns that such disorder would produce. ${ }^{4}$ Previously reported Rietveld refinement of IPC- 6 shows that the average structure is a disordered arrangement of $50 \%$ OKO connections and 50\% PCR, but provides no information about how the different layers are stacked relative to each other (as Rietveld analysis only gives a bulk average). ${ }^{4}$ To address this issue we prepared simulated crystallites with different possible stacking sequences using DISCUS ${ }^{13,14}$ and calculated their PXRD patterns to compare with experiment (Figure 4) as already demonstrated for $\mathrm{AEI} / \mathrm{CHA}$ intergrowth structure. ${ }^{14,15}$ 

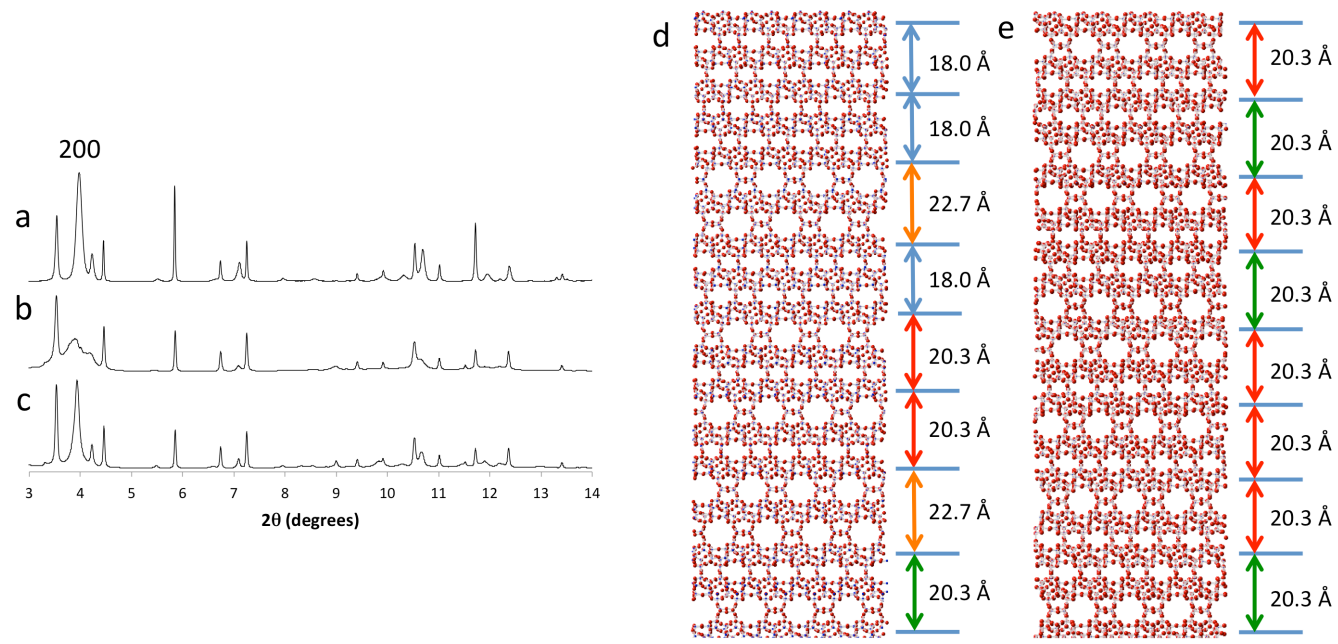

Figure 4. Experimental and simulated XRD patterns and the structure of IPC-6. The experimental synchrotron PXRD pattern for IPC-6 is shown in (a). (b) shows the calculated PXRD patterns for a random arrangement of 50\% OKO and 50\% PCR type linkages between the layers and (c) an arrangement where there is strict retention of the $20.3 \AA$ An unit cell (see discussion). (d) shows a portion of one possible random arrangement of layers used to calculate the pattern shown in (b) and (e) shows a portion of a layer stacking model used to calculate the pattern shown in (c). The coloured arrows indicate the distances in the model, a green arrow indicates layers with contiguous OKO/PCR connections, red arrows show PCR/OKO connections, orange arrows indicate OKO/OKO and blue arrows show PCR/PCR connections. The 200 reflection is marked in the XRD patterns for convenience.

The most obvious possibility for the IPC- 6 structure is a random intergrowth between the IPC-4 and IPC-2 structures. To model this we simulated crystallites that contained $100 \%$ of PCR-type linkages (the pure IPC- 4 framework) and then randomly replaced layers of PCR links with OKO links. PXRD patterns were calculated for different theoretical compositions ranging from 100\% PCR linkages (IPC-4) through to $100 \%$ OKO linkages (IPC-2). The pattern for $50 \%$ PCR-linked layers is one possible structure for IPC- 6 that would be consistent with the average Rietveld refinement result. The calculated XRD pattern for this model is shown in Figure 4b. How this model was derived and the full set of powder patterns for all simulated crystal compositions is shown in the supplementary material, Figures S2 \& S3. However, it is extremely clear by comparison with the experimental PXRD pattern (Figures 4 a) that the IPC-2/IPC-4 intergrowth model does not accurately describe the structure of IPC6. The 200 peak in the experimental IPC-6 pattern is sharp, while that in the calculated pattern is significantly broader. The explanation for this is relatively straightforward. To have sharp h00 peaks, where h corresponds to the interlayer direction, the crystallites must have a consistent $d$-spacing in this direction to ensure constructive interference at only one scattering angle. If the $d$ spacing in the crystallites varies, then constructive interference will occur at different scattering angles leading to a broadening of peaks associated with the interlayer direction. 
The best structural model for IPC- 6 must, therefore, have a d-spacing that is consistent throughout the crystallites. The only way to achieve this is to have a nonrandom combination of IPC-2 and IPC-4 layer connections and to do this we must define a unit cell that consists of exactly one PCR- and one OKO-type connection. The repeating $d_{200}$ for this arrangement will be invariant (and so the 200 reflection will remain sharp) as long as the repeat unit retains the two components. However, since the positions of the diffraction maxima only depend on the d-spacing (Bragg's law), it does not matter which of the two possible orientations of the unit cell are taken (this would, of course, affect the intensity of a reflection, but not its position). Figure 4e shows this schematically with the two orientations shown as different coloured arrows - one orientation of the PCR/OKO is shown as a red arrow while a green arrow shows the opposite OKO/PCR orientation. How this model was derived and the full set of powder patterns for all simulated crystal compositions is shown in the supplementary material, Figures $4 \& 5$. Models for crystallites with this type of structure now give calculated PXRD patterns that retain the sharp 200 reflection and closely match experiment (Figure 4c). The importance of this is that the real structure of IPC- 6 cannot be described in terms of any combination or intergrowth of IPC-2 and IPC-4, but must be a distinct zeolite structure. The main difference between the two models is that there is a relatively high probability of three (or more) successive layer-connections being of the same type (i.e., OKO/OKO/OKO...) in the IPC-2/IPC-4 intergrowth model (Figure 4d) whereas in the correct IPC-6 model the chances of ever having more than two of the same type of connection next to each other is approximately zero (Figure 4e). The 200 reflection for IPC-6 is slightly broader than the other peaks in the pattern indicating that there is a small degree of faulting in the material. This is most likely because the ratio of OKO to PCR linkages is not exactly $1: 1$. Since this ratio changes over time layers as successively become reconnected, it is not surprising that we do not hit exactly a 1:1 ratio. Such faults are visible in the TEM images of IPC- $6 .{ }^{4}$

The only remaining question is how does IPC- 6 form? One can see from the in situ studies and the mechanism shown in Figures 1 and 2 that the precursor to IPC- 6 (IPC-6P) is formed from IPC-1P after partial rearrangement. So why does the IPC-6 structure contain so few occurrences of three successive linkages of the same type? To explain this we take note of the beautiful work of Sartbaeva, Dawson and coworkers who showed that having some 'flexibility' is an important criterion in determining whether a zeolite can be successfully prepared. ${ }^{16,17}$ Figure 5 shows how this concept can be applied to explain why the IPC- 6 structure forms. 


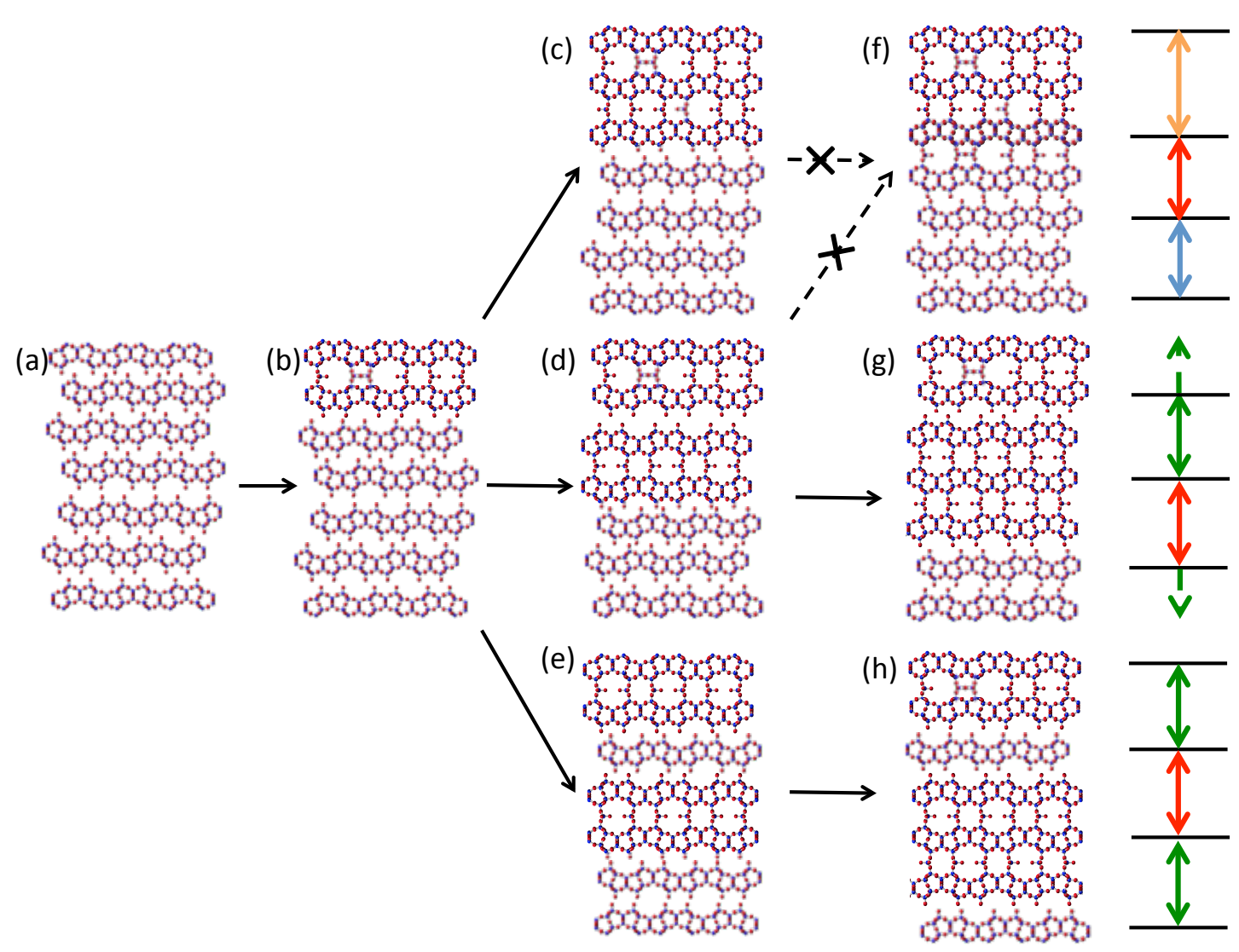

Figure 5. Why does the IPC-6 structure form? The possibilities for sequential rearrangement and reconnection of layers during the ADOR process. Starting from IPC$1 \mathrm{P},(\mathrm{a})$, the first stage is the migration of silica to reconnect one pair of layers (forming one OKO-type linkage). Of the six equally likely places where this could happen only one is shown in (b). The distance between these two layers is then fixed at that found for IPC-2P as the layers are connected by the rearrangement. Over time, the next set of layers can be connected. This can happen between any of the remaining pairs of layers, again fixing their interlayer distance at that found in IPC-2P. Three of these possibilities are shown in (c), (d) and (e). However, as the ADOR process proceeds further there is now a restriction on the next set of layers that can be connected. There is a lower barrier to reconnection of layers that are not adjacent to layers that are already connected, and so it is these connections that happen first. This means that, until it is unavoidable, there are never three contiguous IPC-2P connections. This means that structures (g) and (h) form in preference to structure (f). The consequence of this is that the d-spacing in (g) and $(\mathrm{h})$ is always the same, leading to sharp peaks in the PXRD pattern. In structure (f) the d-spacing would vary, which would lead to the broadened 200 reflection. The colour coding for the $\mathrm{d}$-spacing is as for Figure 4.

Starting from structure shown in Figure $5 a$, the six linkages are all of the same PCR type, as calcination would lead to the IPC-4 (PCR) structure. We can describe this crystallite in terms of its linkages as PCR/PCR/PCR/PCR/PCR/PCR. Under acidic conditions the organisation/rearrangement step in the ADOR process proceeds and layers begin to be connected together by the rearrangement to form OKO-type linkages. In the first instance each of the six possible linkages are equally likely, with one possible arrangement (OKO/PCR/PCR/PCR/PCR/PCR) shown in Figure 5b. Once 
two layers have been connected by OKO-type linkages another set of linkages can begin to form, either between these and an adjacent layer, or between other separate layers. From looking at the IPC- 6 model in Figure $4 \mathrm{e}$ we assume that both these possibilities are approximately equally likely, so that the three possibilities shown in Figures $5 c, d$ and e are all possible. These three arrangements are respectively; OKO/OKO/PCR/PCR/PCR/PCR, OKO/PCR/OKO/PCR/PCR/PCR and $\mathrm{OKO} / \mathrm{PCR} / \mathrm{PCR} / \mathrm{OKO} / \mathrm{PCR} / \mathrm{PCR}$. There are two other equally likely arrangements that are not shown. As yet, all possibilities have been equally likely. However, as the rearrangement process proceeds further the restriction on the unit cell determined from the X-ray diffraction of IPC- 6 means that three contiguous OKO linkages are less favoured than other arrangements. Therefore, Figure $5 f$, which has the OKO/OKO/OKO/PCR/PCR/PCR arrangement is much less likely to occur than the other possible arrangements OKO/PCR/OKO/OKO/PCR/PCR (Figure $5 \mathrm{~g}$ ) and OKO/PCR/PCR/OKO/OKO/PCR (Figure $5 \mathrm{~h}$ ). We believe this restriction occurs because as more and more contiguous layers are connected there is a reduction in flexibility since the layers become tied together, reducing the degrees of freedom in the system and making it harder to intercalate extra silicon and reconnect the layers. This means the probability of more than two contiguous OKO connections is significantly reduced compared to the other possibilities. We know that the probability of connecting further layers is not reduced to zero as, if left for a longer time, the material will continue to rearrange until eventually IPC-2P is formed, with $100 \%$ OKO-type connections. Clearly, the picture of the process shown in Figure 5 is a simplification, which is likely to be a dynamic process (as shown by the NMR measurements above). It does, however, offer a possible explanation of why the structure of IPC- 6 forms.

Given the ADOR mechanism described above, one could imagine that a rather less controlled organisation step may be a way of preparing a more disordered material. Indeed, that is the case - completing the ADOR process with rapid heating in a microwave does give a final product that has severely broadened 200 diffraction peaks (see the supplementary material, Figure 6), indicating that more examples of three contiguous OKO linkages are found in this material than in IPC-6. This material can no longer be described as IPC- 6 , but is much more akin to an intergrowth between the IPC- 2 and IPC- 4 structures.

\section{Conclusions}

By careful in situ MAS NMR and XRD studies of the ADOR process under different conditions we can elucidate the general mechanistic steps by which the process proceeds with time. The experiments clearly show that under high acidity conditions a silica rearrangement occurs that successively 'ties' layers together, and that this has profound effects on the outcome of the ADOR process as a whole. Importantly, the mechanism can be used to explain why IPC- 6 forms with such an unusual and unexpected structure, proving that it cannot be described as an intergrowth of IPC-2 and IPC-4, but rather as a new zeolite structure in its own right. 


\section{Materials and Methods}

\section{Synthesis of ${ }^{29}$ Si-enriched UTL}

A gel with the following molar composition $0.8 \mathrm{SiO}_{2}: 0.4 \mathrm{GeO}_{2}: 0.4 \mathrm{ROH}: 38 \mathrm{H}_{2} \mathrm{O}$ was prepared by dissolving amorphous germanium dioxide $(0.609 \mathrm{~g}, 5.82 \mathrm{mmol}, 99.999 \%$ Acros) in a $0.584 \mathrm{M}$ solution of the SDA, (6R,10S)-6,10-dimethyl-5azoniaspiro[4,5]decane hydroxide $(10 \mathrm{~mL}, 5.84 \mathrm{mmol})$. Tetraethyl orthosilicate ( $2.094 \mathrm{~g}, 10.05 \mathrm{mmol}, 98 \%$ Aldrich) was then added dropwise over 5 minutes and, to enrich the final zeolite, ${ }^{29}$ Si-enriched tetraethyl orthosilicate $(0.333 \mathrm{~g}, 1.59 \mathrm{mmol}$, $>99$ at\% ${ }^{29}$ Si CortecNet) was added. This was stirred at room temperature for 2 hours to allow hydrolysis of the tetraethyl orthosilicate and the removal of ethanol generated from this process. The solution was then transferred to a Teflon-lined autoclave $\left(23 \mathrm{~mL}\right.$, Parr Instruments) and heated at $175^{\circ} \mathrm{C}$ for 13 days under static conditions. The solid was recovered by filtration, washed with copious amounts of distilled water and air dried overnight.

Calcination of the as-synthesised material was carried out to remove the SDA. The zeolite was heated to $575^{\circ} \mathrm{C}$ at a rate of $1{ }^{\circ} \mathrm{C} \mathrm{min}{ }^{-1}$, held at $575{ }^{\circ} \mathrm{C}$ for 6 hours and cooled to room temperature at a rate of $2{ }^{\circ} \mathrm{C} \mathrm{min}^{-1}$ under an atmosphere of air.

\section{Samples for ex situ XRD and NMR studies}

The starting Ge-UTL was synthesised according to a previously reported procedure. ${ }^{4}$ Samples were synthesised by hydrolysing $250 \mathrm{mg}$ of starting Ge-UTL in $40 \mathrm{~mL}$ of either $1.5 \mathrm{M}$ or $5 \mathrm{M} \mathrm{HCl}$ for variable reaction times from 10 minutes to 48 hours at $95^{\circ} \mathrm{C}$. Selected samples were then analysed by solid-state NMR in their hydrolysed form. For the evaluation of the interlayer spacing by ex situ XRD hydrolysed samples were calcined to their reassembled form. Typically the zeolite was heated to $575{ }^{\circ} \mathrm{C}$ at a rate of $1^{\circ} \mathrm{C} \mathrm{min}$, held for 6 hours at $575^{\circ} \mathrm{C}$ and cooled to room temperature at a rate of $2.5^{\circ} \mathrm{C} \mathrm{min}{ }^{-1}$ under an atmosphere of air.

\section{In situ MAS NMR studies of disassembly and organisation}

Solid-state NMR spectra were acquired on a Bruker Avance III spectrometer, equipped with a 9.4 T wide-bore superconducting magnet, operating at a Larmor frequency of $79.459 \mathrm{MHz}$ for ${ }^{29} \mathrm{Si}$. For ex situ measurements, samples were packed in $4 \mathrm{~mm} \mathrm{ZrO}$ rotors and rotated at a MAS rate of $10 \mathrm{kHz}$, using a conventional $4 \mathrm{~mm}$ $\mathrm{HX}$ probe. ${ }^{29} \mathrm{Si}$ MAS NMR spectra were acquired with radiofrequency nutation rates of 71 to $83 \mathrm{kHz}$. Signal averaging was carried out for between 240 and 632 transients with a recycle interval of $120 \mathrm{~s}$. The $Q^{4} / Q^{3}$ ratio was determined from analytical integration using $\mathrm{DMFit}^{18}$ with a confidence interval on data points of \pm 0.4 estimated from multiple fits. For the in situ MAS NMR experiments, Ge-UTL (20 mg, $18 \%{ }^{29}$ Si-enriched) was mixed with 5-10 mg (approximately $10 \mathrm{~mL}$ ) of either distilled water or freshly-prepared $6 \mathrm{M} \mathrm{HCl}$ in disposable PTFE "HR MAS" inserts (Bruker). The inserts were sealed and placed in $4 \mathrm{~mm} \mathrm{ZrO}$ rotors and rotated at a MAS rate of 5 $\mathrm{kHz}$, using a conventional $4 \mathrm{~mm} \mathrm{HX}$ probe. ${ }^{29} \mathrm{Si}$ MAS NMR spectra were acquired with a radiofrequency nutation rate of $83 \mathrm{kHz}$. Signal averaging was carried out for between 64 and 256 transients with a recycle interval of $30 \mathrm{~s}$. In all cases, chemical shifts are shown in ppm relative to $\mathrm{Si}\left(\mathrm{CH}_{3}\right)_{4}$, using the $\mathrm{OSi}\left(\mathrm{CH}_{3}\right)_{3}$ resonance of 
octakis(trimethylsiloxy)silsesquioxane $(\mathrm{Q} 8 \mathrm{M} 8)(\delta=11.5 \mathrm{ppm})$ as a secondary solid reference.

\section{In-situ synchrotron XRD studies of reassembly}

The in-situ powder diffraction measurements were obtained at beam line 111 at Diamond Synchrotron Source, U.K. A fully polarised beam of wavelength $0.708597 \AA$ was used. Samples (IPC-1P, IPC-2P or IPC-6P) were packed in air at ambient pressure inside a rotating borosilicate glass tube of $0.5 \mathrm{~mm}$ outer diameter and heated from 30 to $700{ }^{\circ} \mathrm{C}$. Data was collected using a PSD Mythen2 detector in Debye-Sherrer geometry, collecting every $25^{\circ} \mathrm{C}$ from $30-330^{\circ} \mathrm{C}$ and every $10{ }^{\circ} \mathrm{C}$ thereafter. Two 20 $s$ collections were obtained at each temperature (from $2.25-90^{\circ} 2 \theta$ ) and these were summed to give the final patterns.

\section{Modelling of IPC-6 PXRD}

The PXRD patterns derived from the crystal structures of IPC-4 (PCR framework), OKO (IPC-2) and IPC- 6 were modelled using the DISCUS ${ }^{13,14}$ program, operating in stacking fault mode. The basic structural units used were: IPC-4 based layer (type A) and IPC-2 based layer (type B). The difference between the two-layer units is the presence of an extra S4R unit in layer type B. The parent structure of IPC-4 can be expressed as a perfect stacking of AAA... layers, whereas the stacking of BBB... layers gives a perfect IPC-2 structure. To ensure good averaging of possible structures, each modelled crystallite contained 200 layers. The simulated PXRD patterns were then calculated from the average of 50 such crystallites, each of which had a different stacking order. A wavelength of $0.708597 \AA$ was used in all calculations to match the experimental synchrotron X-ray data collection shown in Figure 4c.

Two fundamentally different stacking arrangements were built. Firstly starting from IPC-4 (PCR/PCR/PCR...) random replacement of 50\% PCR with the same number of OKO-type layers gave a crystallite with 200 layers. This was repeated for 50 crystallites and the PXRD pattern calculated is shown in Figure $4 \mathrm{~b}$. Secondly, a series of PXRD patterns for random stacking sequences of pairs of layers containing strictly one PCR-type and one OKO-type layer were calculated. Starting from a strict ordered $\mathrm{PCR} / \mathrm{OKO} / \mathrm{PCR} / \mathrm{OKO} / \mathrm{PCR} / \mathrm{OKO}$... stacking sequence random pairs of $\mathrm{PCR} / \mathrm{OKO}$ layers were swapped to OKO/PCR until $50 \%$ of the pairs were oriented PCR/OKO and $50 \%$ OKO/PCR. The PXRD pattern calculated from 50 such simulated crystallites is shown in Figure 4c.

\section{References}

1. Roth, W.J., et al. A family of zeolites with controlled pore size prepared using a top-down method. Nature Chem. 5, 628-633 (2013).

2. Eliasova, P., et al. The ADOR mechanism for the synthesis of new zeolites. Chem. Soc. Rev. 44, 7177-7206 (2015).

3. Mazur, M., et al. Synthesis of 'unfeasible' zeolites. Nature Chem. 8, 58-62 (2016).

4. Wheatley, P.S. et al. Zeolites with continuously tuneable porosity Angew. Chem. Int. Ed. 53, 13210-13214 (2014). 
5. Morris, R.E. \& Cejka, J. Exploiting chemically selective weakness in solids as a route to new porous materials. Nature Chem. 7, 381-388 (2015).

6. Paillaud, J.L., Harbuzaru, B., Patarin, J. \& Bats, N. Extra-large-pore zeolites with two-dimensional channels formed by 14 and 12 rings. Science 304, 990992 (2004).

7. Corma, A., Diaz-Cabanas, M.J., Rey, F., Nicolooulas, S. \& Boulahya, K. ITQ-15: The first ultralarge pore zeolite with a bi-directional pore system formed by intersecting 14- and 12-ring channels, and its catalytic implications. Chem. Comm. 1356-1357 (2004).

8. Corma, A. Rey, F., Valencia, S., Jorda, J.L. \& Rius, J. Nature Mater. 2, 493497(2003).

9. Chlubna-Eliasova, P., et al. The Assembly-Disassembly-OrganizationReassembly Mechanism for 3D-2D-3D Transformation of Germanosilicate IWW Zeolite. Angew. Chem. Int. Ed. 53, 7048-7052 (2014).

10. Lorgouilloux, Y., et al. IM-17: a new zeolitic material, synthesis and structure elucidation from electron diffraction ADT data and Rietveld analysis. RSC Adv. 4, 19440-19449 (2014).

11. Kasneryk, V., et al. Expansion of the ADOR strategy for the synthesis of new zeolites: The synthesis of IPC-12 from zeolite UOV Angew. Chem. Int. Ed. Submitted for Publication.

12. Roth, W.J., et al. Postsynthesis Transformation of Three-Dimensional Framework into a Lamellar Zeolite with Modifiable Architecture. J. Am. Chem. Soc. 133, 6130-6133 (2011).

13. Proffen, T. \& Neder, R.B. DISCUS: A program for diffuse scattering and defectstructure simulation. J. Appl. Crystallogr. 30, 171-175 (1997).

14. Slawinski, W.A., Wragg, D.S., Akporiyae, D. \& Fjellvag, H Intergrowth structure modelling in silicoaluminophosphate SAPO-18/34 family Micropor. Mesopor. Mater. 195, 311-318 (2014).

15. Smith, R. L., et al. Nanoporous Intergrowths: How Crystal Growth Dictates Phase Composition and Hierarchical Structure in the CHA/AEI System. Chem. Mater. 27, 4205-4215 (2015).

16. Sartbaeva, A., Wells, S.A., Treacy, M.M.J. \& Thorpe, M.F. The flexibility window in zeolites. Nature Mater. 5, 962-965 (2006).

17. Dawson, C. J., Kapko, V., Thorpe, M. F., Foster, M. D. \& Treacy, M. M. J. Flexibility as an indicator of feasibility of zeolite frameworks. J. Phys. Chem C 116, 16175-16181 (2012).

18. Massiot, D. et al. Modelling one- and two-dimensional solid-state NMR spectra Magn. Reson. Chem. 40, 70-76 (2002).

\section{Acknowledgements}

R.E.M. and M.N. thank the Royal Society and the E.P.S.R.C. (Grants EP/L014475/1, EP/K025112/1 and EP/K005499/1) for funding work in this area. R.E.M. and J.Č. acknowledge the Czech Science Foundation for the project P106/12/G015 and OP VVV "Excellent Research Teams", project No. 
CZ.02.1.01/0.0/0.0/15_003/0000417 - CUCAM. S.E.A. would like to thank the ERC (EU FP7 Consolidator Grant 614290 "EXONMR") and the Royal Society and Wolfson Foundation for a merit award. We thank Chiu Tang for his assistance with the synchrotron PXRD work carried out at the Diamond Light Source, UK. The UK 850 $\mathrm{MHz}$ solid-state NMR Facility used in this research was funded by EPSRC and BBSRC (contract reference PR140003), as well as the University of Warwick including via part funding through Birmingham Science City Advanced Materials Projects 1 and 2 supported by Advantage West Midlands (AWM) and the European Regional Development Fund (ERDF). Collaborative assistance from the $850 \mathrm{MHz}$ Facility Manager (Dinu luga, University of Warwick) is acknowledged. W.A.S. and D.S.W. acknowledge the Research Council of Norway and NOTUR are acknowledged for providing the computer time at the Norwegian supercomputer facilities (under the project number NN2875k).

\section{Additional information}

Reprints and permissions information is available online at www.nature.com/reprints. Correspondence and requests for information should be addressed to R.E.M. (rem1@st-and.ac.uk).

Author Contributions. P.S.W. designed the route and synthesised the enriched UTL samples. S.A.M., P.S.W. and D.S.F. completed the in situ PXRD. Y.T. and M.N. completed the initial ADOR synthesis. W.A.S. and D.S.W. simulated the PXRD using computational approaches. G.P.M.B., D.M.D. and S.E.A. designed and completed the NMR experiments. J. Č. collaborated on the design of the project, and R.E.M. and S.E.A coordinated the project and wrote the paper.

\section{Competing financial interests}

The authors declare no competing financial interests. 